\title{
Substructure Formation around Fatigue Cracks and Its Role in the Propagation of Fatigue Cracks in Aluminium*
}

\author{
By Tsugio Ogura** and Seiichi Karashima***
}

\begin{abstract}
Polycrystalline aluminium specimens of $93.93 \%$ purity were fatigued in alternate tension and compression with zero mean stress. Substructures near the tip of fatigue cracks ware exanined at various stages of crack propagation using an $\mathrm{X}$-ray micro-beam technique. The propagation rate of the fatigue crack was also measured by a replicating method. The main results obtained are as follows.

(1) Regardless of the intensity of fatigue stress and the length of fatigus cracks, there exists a plastic zone around a fatigue crack in aluminium.

(2) A well-defined substructure characterized by the following three features is observed in the plastic zone : (i) heavily fragmented subgrains $\left(1 \sim 2 \mu\right.$ in diameter), (ii) very low excess dislocation density within a subgrain (about $\left.10^{8} / \mathrm{cm}^{2}\right)$, and (iii) sharply defined sub-boundaries probably consisting of regular and simple arrays of dislocations. These characteristics are considered to be due to the generation of vacancies in large quantities and also to the stress concentration at the tip of a fatigue crack.

(3) Two kinds of plastic zones, one observed by an X-ray micro-beam technique and the other in the studies made from a macroscopic point of view, are concluded to be the same.

(4) The propagation rate of fatigue crack, $d l / d N$, can be related to the size of the plastic zone, $Z_{p}$, by an equation,

$$
d l / d N=A Z_{p}^{m},
$$

where $A$ is a constant independent of fatigue stress and $m$ an exponent of about 2 .

(5) Intimate relations between the propagation rate of fatigue crack and some structural parameters of the plastic zone were expla ined qualitatively by a vacancy absorption mechanism.
\end{abstract}

(Received March 9, 1972)

\section{Introduction}

It has been suggested that substructure formation plays an important role in fatigue fracture. This suggestion has been supported by the following results which have recently been obtained by transmission electron microscopy ${ }^{(1) \sim(6)}$ and X-ray observations ${ }^{(4)}(7) \sim$ (10). Around a fatigue crack in some $f c c$ metals and alloys there exists a plastic zone in which a well-defined substructure is formed. According to the study on aluminium $^{(11)}$ fatigued by alternating tension and compression, the propagation rate of fatigue crack is the faster, the formation of substructure near the crack tip and/or in the matrix is the more remarkable.

Several suggestions have so far been made on the role of substructure formation in fatigue crack propagation $^{(7)(12) \sim(14)}$. However, none of them has directly been verified experimentally. Therefore, it can be said that many problems are left unsolved on this matter and that fundamental and systematic experiments are necessary to settle the question.

* This is a summation of the reports which were originally published in Japanese in J. Japan Inst. Metals, 34 (1970), 739 and 888.

** The Research Institute for Iron, Steel and Other Metals, Tohoku University, Sendai, Japan.

*** Department of Materials Science, Faculty of Engineering, Tohoku University, Sondai, Japan.

(1) R. N. Wilson and P. J. E. Forsyth : J. Inst. Metals, 87 (1959), 912.

(2) S. Karashima, H. Oikawa, and T.Ogura : J. Japan Inst. Metals, 31 (1967), 674.

(3) J. C. Grosskreutz and G. G. Shaw : Fatigue Crack Propagation, ASTM STP 415, (1967), p. 226.

(4) S. Karashima, H. Oikawa, and T.Ogura : Trans. JM, 9 (1968), 205.
In the present study, substructure observation was made at various stages of fatigue crack propagation under several fatigue stresses, in order to obtain a fundamental knowledge for clarifying the mechanism of fatigue fracture. From the above observation, the effects of fatigue stress and fatigue crack length, i. i.e. the effects of stress concentration at the crack tip, on the substructure formation were first examined. Next, some characteristics of the plastic zone around fatigue cracks revealed by $\mathrm{X}$-ray observations were compared with those found in the studies made from a macroscopic point of view. Finally, the role of substructure formation in fatigue crack propagation was discussed on the basis of a model proposed by the present authors ${ }^{(15)}$.

\section{Experimental Methods}

Aluminium of $99.99 \%$ purity was melt in air and cold-rolled to a plate of $2 \mathrm{~mm}$ thickness. The specimens shown in Fig. 1 were cut from the plate and annealed at $235^{\circ} \mathrm{C}$ for 7 days. The mean grain diameter and the yield stress of the specimens after the

(5) M. Klesnil and P. Lukás̆ : Phil. Mag., 17 (1968), 1295.

(6) P. Lukáš, M. Klesnil, and R. Fiedler : ibid., 18 (1969), 799.

(7) J. Holden : Phil. Mag., 6 (1961), 547.

(8) J. C. Grosskreutz and P. Waldow : Acta Met., 11 (1963), 717.

(9) S. Taira and K. Hayashi : Bulletin of JSME, 9 (1966), 627.

(10) S. Karashima, H. Oikawa, and T. Ogura : J. Japan Inst. Metals, 31 (1967), 669.

(11) S. Karashima and T. Ogura : Czech. J. Phys, B 19(1969), 362.

(12) J. C. Grosskreutz : J. Appl. Phys., 33 (1962), 1787.

(13) D. H. Avery and W. A. Backofen : Fracture of Solids, Interscience Pub, (1962), p. 339.

(14) A. J. McEvily and R. C. Boettner : Acta Met., 11 (1963), 725.

(15) T. Ogura and S. Karashima : Trans. JIM, 10 (1969), 292. 

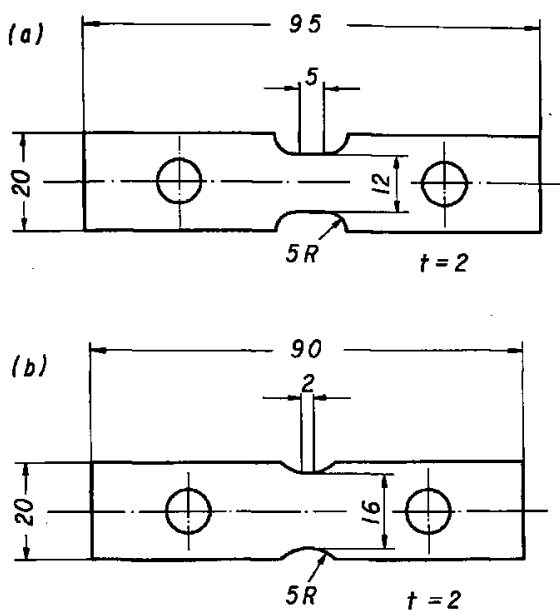

Fig. 1 Fatigue specimens (dimension in $\mathrm{mm}$ ) (a) at low and medium stresses fatigue (b) at high stress fatigue.

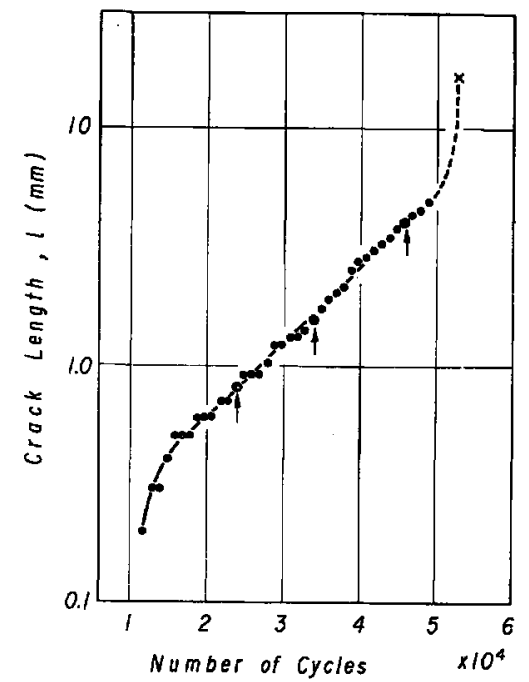

Fig. 2 An example of fatigue crack propagation curves. $\mathrm{X}$-ray observations were done at three stages indicated by arrows (fatigue stress: $2.3 \mathrm{~kg} / \mathrm{mm}^{2}$ )

annealing treatment were about $35 \mu$ and $2.1 \mathrm{~kg} / \mathrm{mm}^{2}$, respectively.

A fatigue machine of the tension-compression type with zero mean stress was employed at a frequency of $1800 \mathrm{cycle} / \mathrm{min}$. The specimens were fatigued in air at stresses of $1.6,1.9$ and $2.3 \mathrm{~kg} / \mathrm{mm}^{2}$. The fatigue tests with the alternating stresses mentioned above are named hereafter low stress fatigue, medium stress fatigue, and high stress fatigue, respectively. Before the fatigue test a notch was made chemically on a side surface of the specimen which was subsequently polished electrolytically. The depth and the radius of curvature at the root of the notch after electropolishing was 0.6 $\mathrm{mm}$ and $0.1 \mathrm{~mm}$, respectively.

An example of the fatigue crack propagation curves obtained in the present study is shown in Fig. 2. The crack length was measured by an optical microscope with an accuracy of $0.01 \mathrm{~mm}$ on plastic replicas which had been obtained from the surface of the specimen set on the machine. For microbeam X-ray observations, the fatigue test was interrupted and the specimen was removed from the machine at three stages indicated by arrows in the figure. Locations of the $\mathrm{X}$-ray observations near the tip of a fatigue crack are illustrated in Fig. 3. Some parameters of the substructure, i.e. the mean subgrain size, $t$, the lattice misorientation within a subgrain, $\alpha$, the relative lattice disorientation of adjacent two subgrains at their sub-boundary, $\beta$, and the total range of lattice misorientations within an original grain, $\alpha_{\text {Total }}$, were determined from back-reflection $\{422\}$-rings obtained by $\mathrm{CuK}_{\alpha}$ radiation.

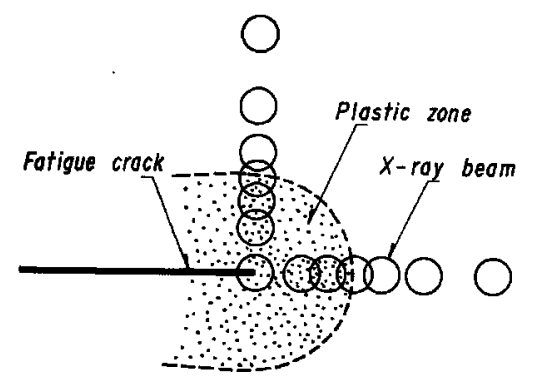

Fig. 3 The hegtion of $X$-ray observations near the tip of a fatigue crack.

\section{Experimental Results and Discussion}

\section{Substrueture formation near the tip of a fatigue crack}

Characteristics of the substructure developed during high stress fatigue will be stated at first. In Figs. 4 6 are shown the results obtained at the stages where crack lengths were $0.8,1.5$ and $3.9 \mathrm{~mm}$, respectively. In the figures $R_{/ /}$is the distance from the crack tip measured in the direction parallel to the crack propagation and $R_{\underline{L}}$ is that measured in the direction normal to it. As clearly seen in the figures, $\alpha_{\text {Total }}$ and $\beta$ increase remarkably near the tip of a fatigue crack. On the contrary $t$ and $\alpha$ decrease in this region. From the above results it can be concluded that decrease in $\alpha$ is mainly caused by decrease in $t$ and that a remarkable increase in $\alpha_{\text {TotaI }}$ is due both to the increase in $\beta$ and to the decrease in $t$, that is, to the formation of new sub-boundaries.
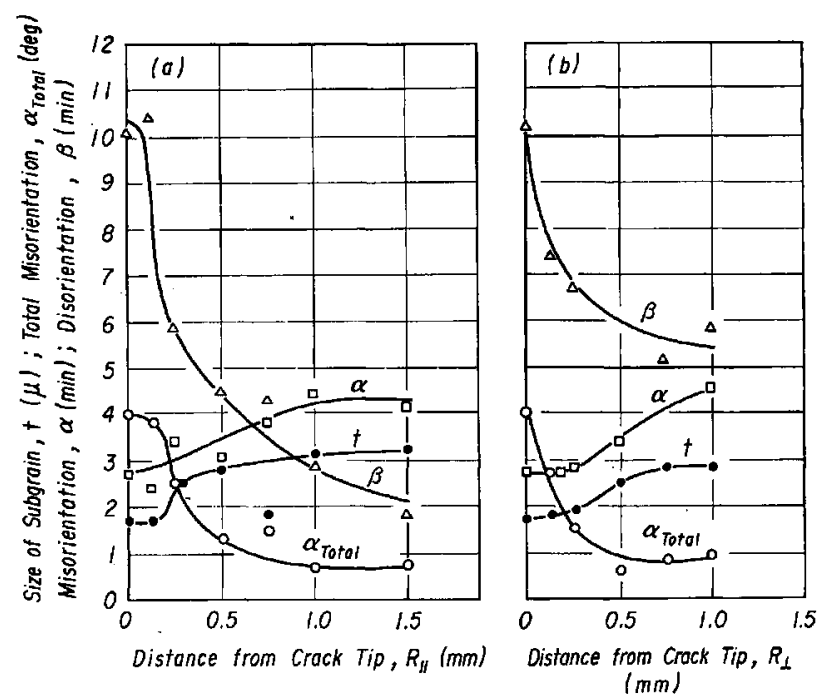

Fig. 4. Change in the substructural parameters with the distance from a crack tip (fatigue stress : $2.3 \mathrm{~kg}$ / $\mathrm{mm}^{2}$, crack length : $0.8 \mathrm{~mm}$ ). 

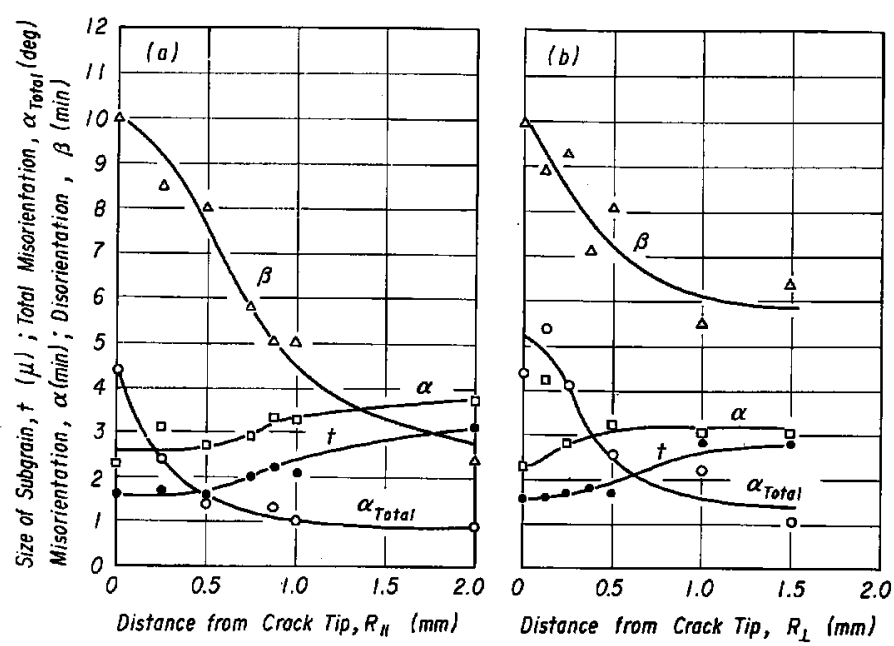

Fig. 5 Change in the substructural parameters with the distance from a crack tip (fatigue stress $: 2.3 \mathrm{~kg}$ / $\mathrm{mm}^{2}$, crack length : $1.5 \mathrm{~mm}$ ).
Factors which may contribute to the formation of the characteristic substructure in the plastic zone are now considered. Effects of stress intensity factor, $K_{I}$, which can be calculated from $\sigma_{f} \sqrt{ } \bar{l}\left(\sigma_{f}\right.$ : fatigue stress and $l$ : crack length), on some structural parameters of the plastic zone are shown in Figs. $7 \sim 10$ for the three stress levels mentioned before. All the parameters are functions of $K_{I} ; t$ and $\alpha$ decrease, and $\beta$ and $\alpha_{\text {Total }}$ increase with increasing $K_{I}$. It thus appears that the stress concentration at the tip of a fatigue crack makes great contributions to the substructure development there.

Change in excess dislocation density within a subgrain, $D_{\alpha}^{* *}$, with the distance from the crack tip is shown in Fig. 11. As seen in the figure, $D_{\alpha}$ is nearly constant (ca. $1.3 \times 10^{8} / \mathrm{cm}^{2}$ ) in the matrix regardless of the crack length. The arrow in the figure shows the position of the interface between matrix and plastic zone which is defined as shown in Fig. 12. Although $D_{\alpha}$ seems to
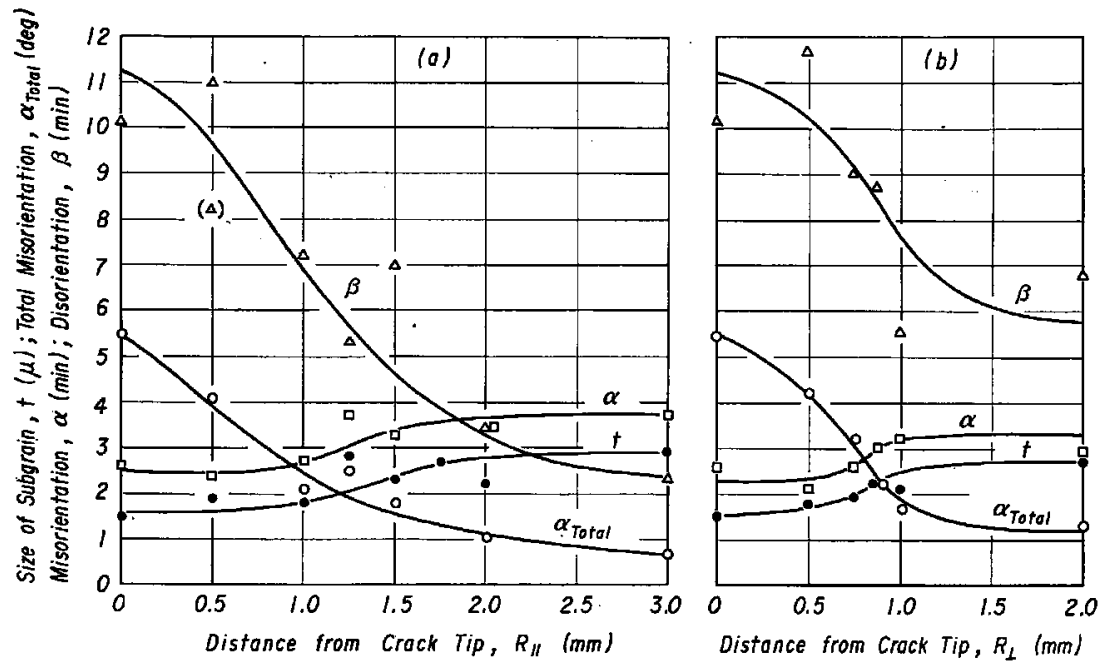

Fig. 6 Change in the substructural parameters with the distance from a crack tip (fatigue stress : $2.3 \mathrm{~kg} / \mathrm{mm}^{2}$, crack length : $3.9 \mathrm{~mm}$ ).

The value of $t$ begins to decrease in the neighbourhood of the point where $\alpha_{\text {Total }}$ begins to increase, and becomes constant $(1 \sim 2 \mu)$ in the region very close to the crack tip. This value is much smaller than that obtained by Hirsch and $\operatorname{Kellar}^{(16)}$ on severely cold rolled aluminium of high purity $(2 \sim 3 \mu)$. On the other hand, $\alpha_{\text {Total }}$ and $\beta$ still continue to increase in this region. These facts show that the increase in $\alpha_{\text {Total }}$ is due largely to the increase in $\beta$. In other words, the formation of new sub-boundaries does not occur so much, because the greater part of dislocations is absorbed into the existing sub-boundaries.

X-ray observations of the specimens subjected to the low stress fatigue and to the medium stress fatigue* gave the results quite similar to those mentioned above. Therefore, it is concluded that in aluminium a plastic zone, within which a well-defined substructure is formed, exists around a fatigue crack regardless of the intensity of fatigue stress and the longth of fatigue cracks.

(16) P. B. Hirsch and J. N. Kellar : Acta Cryst., 5 (1952), 162.

* The results obtained on medium stress fatigue have been published elsewhere(11). increase a little within the plastic zone, its average value at the crack tip $\left(1.7 \times 10^{8} / \mathrm{cm}^{2}\right)$ is about the same as the dislocation density in annealed polycrystalline metals. The result suggests that a marked recovery occurs simultaneously with a dislocation multiplication in the course of fatigue ${ }^{\dagger}$.

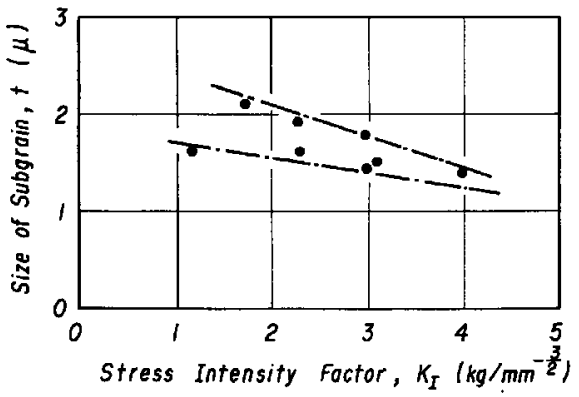

Fig. 7 Change in the subgrain size, $t$, with the stress intensity factor, $\boldsymbol{K}_{\boldsymbol{I}}$.

** $\quad D_{\alpha}$ was calculated from the relation, $D_{\alpha}=\alpha / b t$, where $b$ is the Burgers vector.

$\dagger$ Observations with an electron microscope (see ref. (30)) also show a low density of dislocations within a subgrain formed near fatigue cracks of aluminium. 


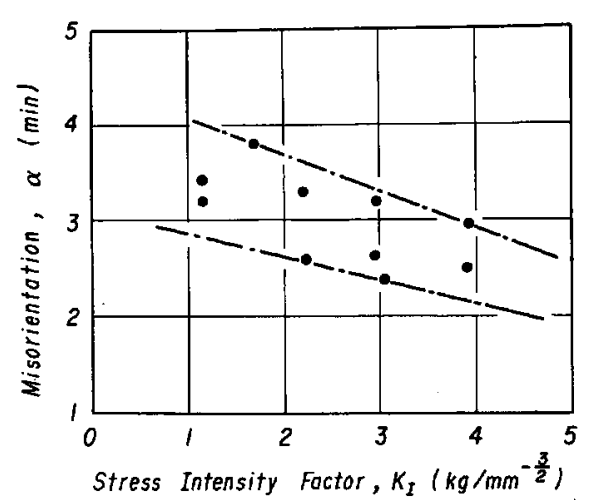

Fig. 8 Change in the misorientation, $\alpha$, with the stress intensity factor, $K_{I}$.

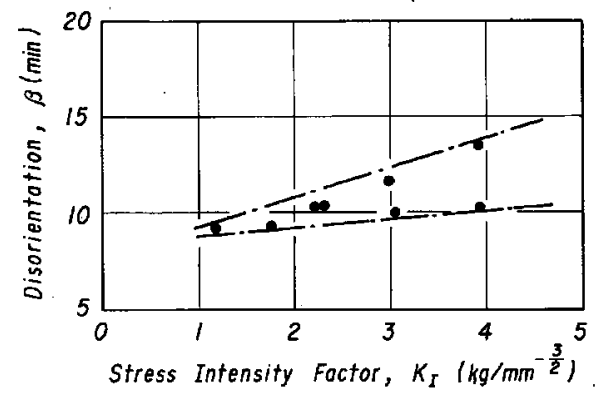

Fig. 9 Change in the disorientation, $\beta$, with the stress intensity factor, $K_{I}$.

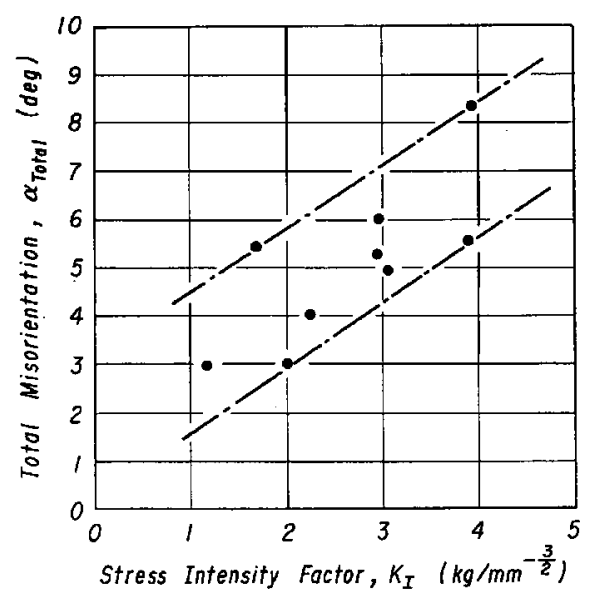

Fig. 10 Change in the total misorientation, $\alpha_{\text {Total, }}$ with the stress intensity factor, $K_{I}$.

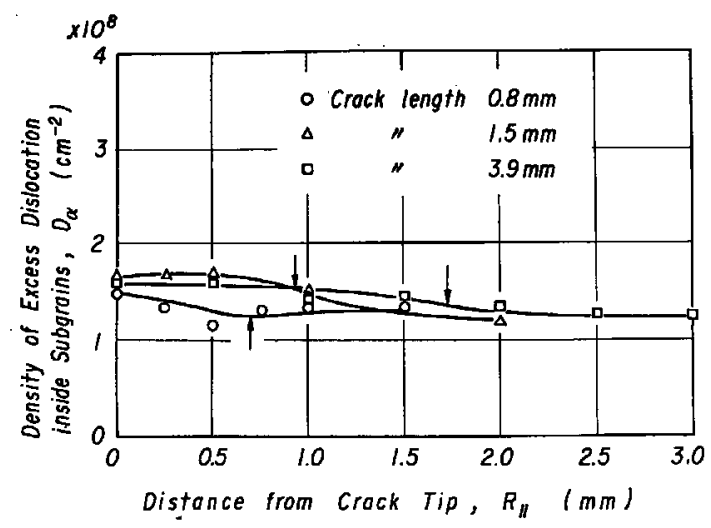

Fig. 11 Change in the excess dislocation density, $D_{a}$, with the distance from a crack tip. Arrow shows the position of the interface between the matrix and the plastic zone which is defined as shown in Fig. 12 (fatigue stress : $2.3 \mathrm{~kg} / \mathrm{mm}^{2}$ ).

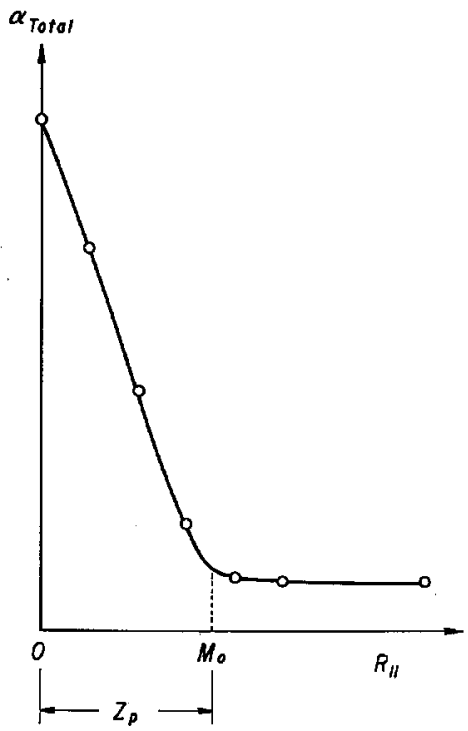

Fig. 12 Schematic representation of the change in the total misorientation, $\alpha_{\text {Total }}$, with the distance from a crack tip. $Z_{p}$ defines the size of the plastic zone.

Characteristics of the substructure formed near the tip of a fatigue crack show themselves also on an appearance of Debye-Scherrer ring. It is well known that sub-boundaries in deformed metals generally consist of many tangled dislocations ${ }^{(17)}$. It is suggested by Hirsch $^{(18)}$ that the background intensity between the diffraction spots in a Debye-Scherrer ring obtained by an X-ray micro-beam technique is due to $\mathrm{X}$-ray beams reflecting from such sub-boundary regions. Comparing the Debye-Scherrer ring obtained near the tip of a fatigue crack with that obtained with unidirectionally strained metal, it can be easily found that the background intensity of the former is remarkably weaker than that of the latter. This fact shows that sub-boundaries formed near a fatigue crack are thinner, the dislocation distribution within them being more regular and simpler due to the dislocation redistribution, i.e. a recovery effect. It is generally accepted on the basis of many experimental results ${ }^{(19)}$ that dislocations, which move to-and-fro during fatigue, cut so many forest dislocations that a multitude of vacancies is generated. The remarkable recovery near a fatigue crack may be caused by these vacanoies.

\section{Some natures of plastic zone formed around a fatigue crack}

In the studies made from a macroscopic point of view, it has also been pointed out that there exists a plastic zone near the tip of a fatigue crack. Such a zone will be called a plastic zone in a macroscopic sense hereafter. Its important role in fatigue crack propagation has frequently been suggested ${ }^{(20)}$. In the following the relation between the plastic zone in a macroscopic sense

(17) P. R. Swann : Electron Microscopy and Strength of Crystals, Interscience Pub., (1963), p. 131.

(18) P. B. Hirsch : Acta Cryst., 5(1952), 168.

(19) For example, N. Thompson : Z. Metallk, 53 (1962), 71.

(20) A. K. Head : Phil. Mag., 44 (1953), 925. 
and the plastic zone detected by the present X-ray observations will be discussed.

A schematic representation of the change in $\alpha_{\text {Total }}$ with the distance from a crack tip is shown in Fig. 12. As seen in the figure, $\alpha_{\text {Total }}$ is nearly constant in the matrix, while it increases rapidly in the plastic zone with decreasing distance from the crack tip. On this curve, we can determine a point at which $\alpha_{\text {rotal }}$ begins to increase remarkably. The distance of the point from the origin (crack tip), $0 M_{0}$, is defined as the size of the plastic zone, $Z_{p}$, in the present study.

Around a fatigue crack there is a region where slip lines are formed densely. An example of such a region (slip-line-concentrating region) is shown in Photo. 1. The size of the slip-line-concentrating region, $Z_{s}$, is determined from the distance from the crack tip measured along the direction of crack propagation. In Fig. 13 , the ratio $Z_{s} / Z_{p}$ is plotted against $Z_{p}$. As seen in the figure, the ratio is nearly equal to unity independently of $Z_{p}$. The result suggests that the plastic zone detected by the $\mathrm{X}$-ray method is probably the same with the slip-line-concentrating region, that is the plastic zone in a macroscopic sense.

The relation between $Z_{p}$ and crack length measured from the notch root, $l$, is shown in Fig. 14 for the three stress levels. The results that $Z_{p}$ increases linearly with $l$ and has a certain positive value at $l=0$ suggest that a plastic zone already exists near the notch root when a

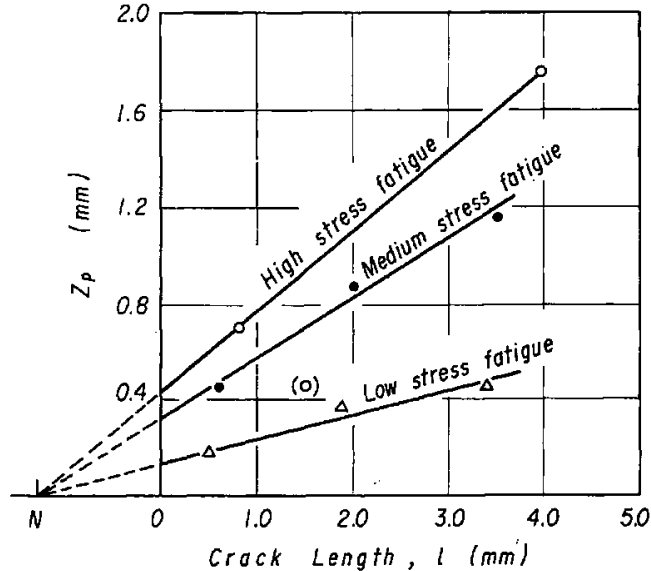

Fig. 14 Variation of the size of the plastic zone with the length of a fatigue crack.

macroscopic fatigue crack is formed. Therefore, it can be said that a notch is equivalent to a fatigue crack of a certain length for the formation of a plastic zone. Extrapolating the straight lines as shown in the figure, a point $\mathrm{N}$ is obtained from their intersection with the abscissa. If $0 \mathrm{~N}$ is assumed as the effective crack length, $l_{0}$, of the notch, $Z_{p}$ is proportional to a modified crack length $l^{\prime}$ defined by $l_{0}+l$. Such a proportionality has also been found in some steels to be a characteristic of the plastic zone in a macroscopic sense ${ }^{(21)}$.

According to the theories of Dugdale ${ }^{(22)}$ and of Bilby

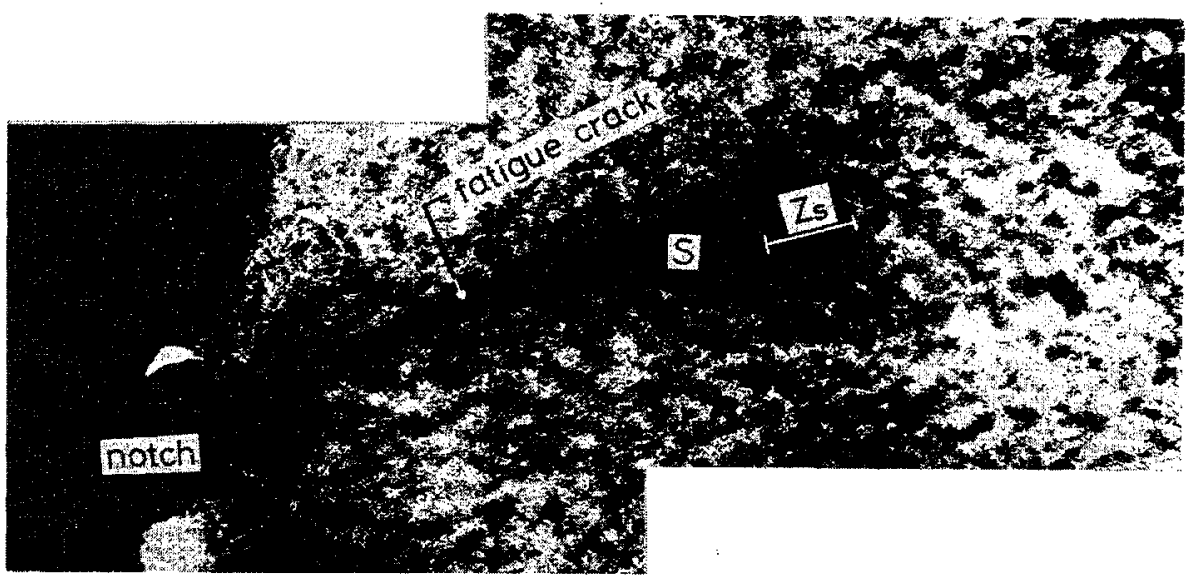

Photo. 1 An example of optical photomicrographs showing slip-line-concentrating region (S) around a fatigue crack.

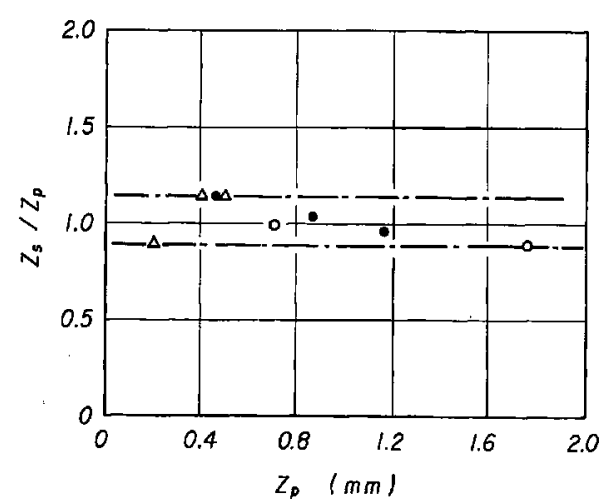

Fig. 13 Ratio of the size of the slip-line-concentrating region to the size of the plastic zone, $Z_{s} / Z_{p}$, versus $Z_{p}$. et al. ${ }^{(28)}$, a quantitative relation between applied stress, $\sigma_{a}$, and $Z_{s}$ in a perfectly plastic solid ${ }^{(24)}$ is expressed by

$$
Z_{s} / l=\sec \left(\pi \sigma_{a} / 2 \sigma_{y}\right)-1
$$

where $l$ is the crack length and $\sigma_{y}$ is the yield stress. Recently Yokobori et al. (25) have examined the formation of the plastic zone around a fatigue crack in some

(21) N.F. Frost and D.S.Dugdale : J.Mech. Phys. Solids, 6 (1958), 92.

(22) D. S. Dugdale : ibid., 8 (1960), 100.

(23) B. A. Bilby, A. H. Cottrell, and K. H. Swinden : Proc. Roy. Soc., A 272 (1963), 304.

(24) S. Timoshenko : Strength of Materials, Part II, Van Nostrand Reinhold, (1956), p. 346.

(25) T. Yokobori, M. Tanaka, H. Hayakawa, T. Yoshimura, and S. Sasahira : Rep. Res. Inst. Strength and Fracture of Materials, Tohoku Univ., 3 (1967), 39. 
steels by an optical microscope and found that the above equation can be applied to the fatigue crack propagation when the peak value of applied alternating stress is used for $\sigma_{a}$. In the case of the plastic zone detected by the $\mathrm{X}$-ray method, it is shown that the above equation is still valid if the modified yield stress* is adopted for $\sigma_{y}{ }^{(26)}$. The fact also shows a close relation between the plastic zone observed in the present experiments with the one in a macroscopic sense.

The relation between $Z_{p}$ and the rate of crack propagation, $d l / d N$, is shown in Fig. 15. As clearly seen

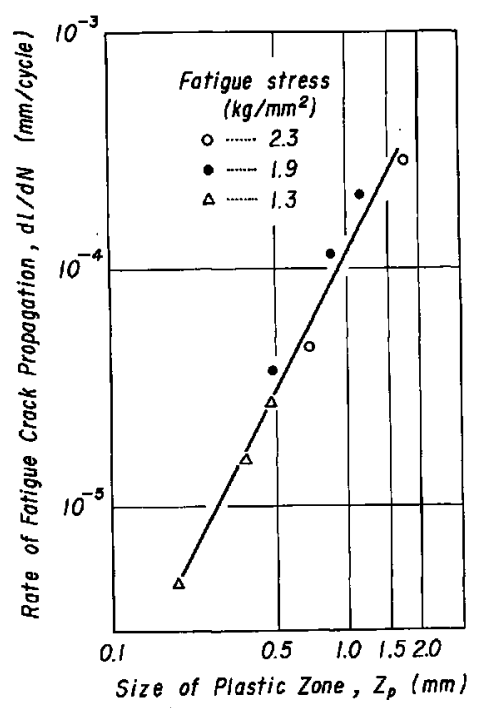

Fig. 15 Change in the propagation rate of a fatigue crack, $d l / d N$, with the size of plastic zone, $Z_{p}$, determined by $\mathrm{X}$-ray observation.

in the figure, $Z_{p}$ is related to $d l / d N$ and is independent of fatigue stress as expressed by the equation,

$$
d l / d N=A Z_{b}^{m},
$$

where $A$ is a constant and $m \cong 2$. Although the physical meaning of this equation is not necessarily clear, the following fact is to be noted. It has been confirmed theoretically ${ }^{(27)}$ and experimentally ${ }^{(14)(28)}$ from a macroscopic point of view that the following relation holds between the stress intensity factor, $K_{I}$, and the propagation rate of fatigue crack.

$$
d l / d N=A^{\prime} K_{I}^{4}
$$

where $A^{\prime}$ is a constant. On the other hand, $K_{I}$ is related to $Z_{s}$ by the equation ${ }^{(29)}$,

$$
Z_{s}=A^{\prime \prime} K_{I}^{2}
$$

where $A^{\prime \prime}$ is a constant. One can derive an equation essentially the same as eq.(2) by combining eq.(3) with eq. (4). The fact is another evidence to confirm that the plastic zone revealed by the present experiments is

* We imagine a perfectly plastic solid which has the fracture strain and the area enclosed by the stress-strain diagram respectively to be the same as those of the aluminium specimen used in the present experiments. The yield stress of the solid is taken as the modified yield stress here.

(26) S. Karashima, H. Oikawa, and T. Ogura : Unpublished work.

(27) P. C. Paris : An Interdisciplinary Approach-Fatigue, Syracuso Univ. Press, (1964), p. 107 the same with that deduced from a macroscopic viewpoint.

\section{Effects of some structural parameters of the plastic zone on the propagation rate of fatigue crack}

In previous papers ${ }^{(15)(30)}$, it was reported that many small voids were found near the tip of a fatigue crack and that these voids grew up to combine with the main crack during tensile deformation in an electron microscope. On the basis of the above experimental results, it was proposed that fatigue cracks in aluminium propagate growing-up and joining-up of these voids during each by fatigue cycle. The growth of the voids was supposed to be caused by absorbing vacancies which were generated in the plastic zone and were driven towards the main crack due to the stress gradient near the crack tip. On the other hand, it is quite possible that the diffusion of vacancies is enhanced by the presence of sub-boundaries. In order to verify the supposition stated above, the effects of the substructure formed at the tip of a fatigue crack on the propagation rate of fatigue crack were examined.

Propagation rates of fatigue crack at high and low stresses are plotted in Figs. 16 19 against four kinds of structural parameters $\left(v, \alpha, \beta\right.$, and $\left.\alpha_{\text {Total }}\right)$ of the plastic zone formed at the tip of a fatigue crack. As seen in the figures, $d l / d N$ increases as $v$ and $\alpha$ decrease and as $\beta$ and $\alpha_{\text {Tota }}$ increase. The relations demonstrated in these figures agree qualitatively with those obtained in medium stress fatigue ${ }^{(11)}$.

As the size of subgrains decreases, that is the fraction of volume occupied by sub-boundaries becomes larger, the number of paths for enhanced diffusion of vacancies may increase. Therefore, the result that $d l / d N$ is

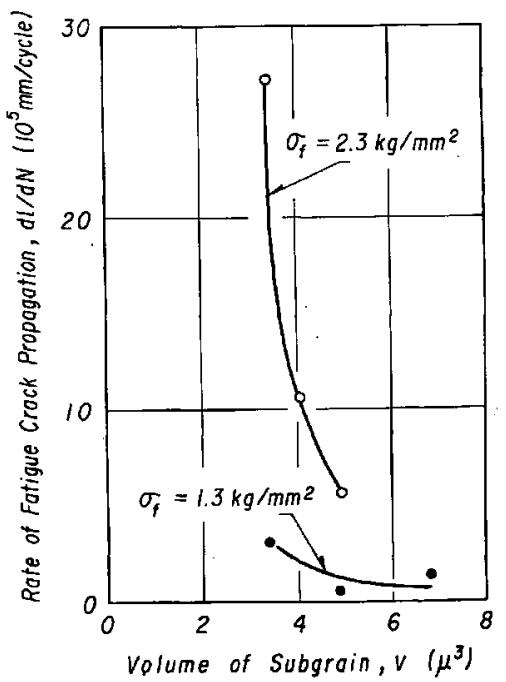

Fig. 16 Change in the propagation rate of a fatigue crack, $d L / d N$, with the volume of subgrains, $v$, at the tip of a fatigue crack.

(28) R. W. Hertzwerg and P.C. Paris : Proc. 1st International Conference on Fracture, Vol. 1, (1965), p. 459.

(29) J.R. Rice : Fatigue Crack Propagation, ASTM STP 415, (1967), p. 247.

(30) T. Ogura and S. Karashima : J. Japan Inst. Metals, 34(1970), 746. 


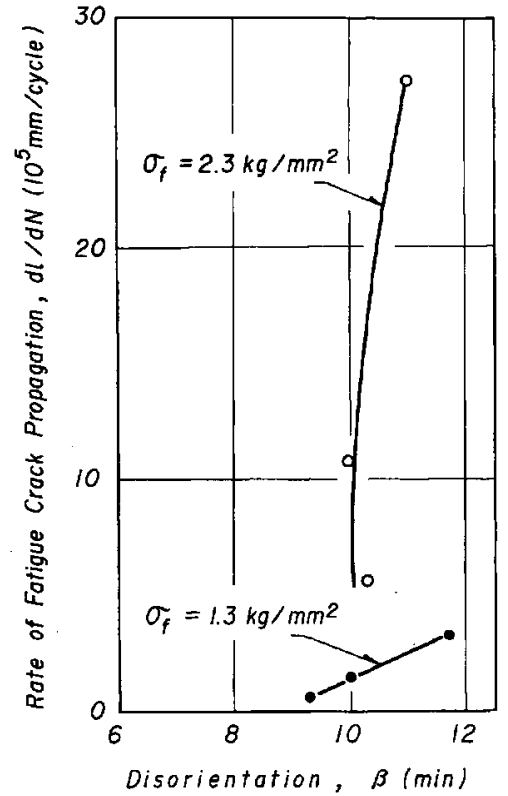

Fig. 17 Change in the propagation rate of a fatigue crack, $d l / d N$, with the disorientation, $\beta$, at the tip of a fatigue crack.

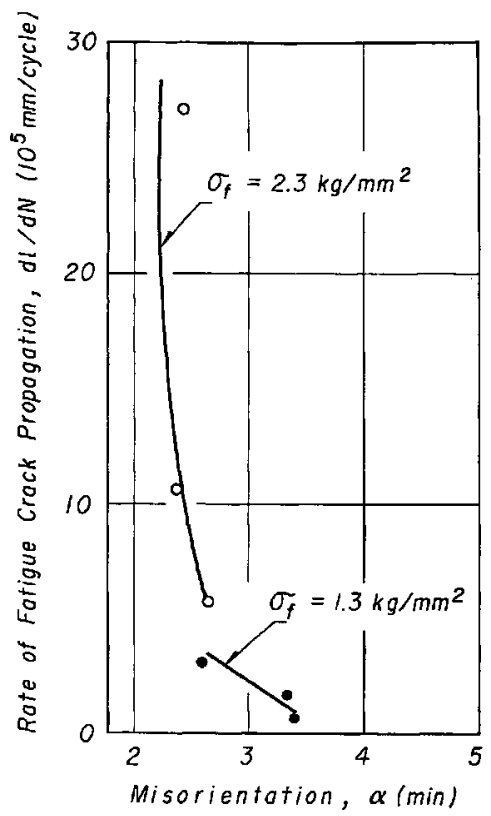

Fig. 18 Change in the propagation rate of a fatigue crack, $d l / d N$, with the misorientation, $\alpha$, at the tip of a fatigue crack.

larger as $v$ becomes smaller seems consistent with the model proposed by the present authors. Similarly, the relation between $\beta$ and $d l / d N$ shown in Fig. 17 can also be explained qualitatively. It is shown in Fig. 11 that $D_{\alpha}(=\alpha / b t)$ at the tip of a crack remains almost constant during crack propagation and therefore $\alpha$ is proportional to $t(=\sqrt{ } \sqrt{v})$. The results explain why the dependences of $d l / d N$ on $\alpha$ and on $v$ are similar to each other. The dependence of $d l / d N$ on $\alpha_{\text {Total }}$ shown in Fig. 19 can easily be understood from the dependences of $d l / d N$ on $v$ and on $\beta$. It is thus confirmed that the vacancy absorption mechanism for fatigue crack propagation is consistent with the experimental results on the relation between propagation rates of fatigue

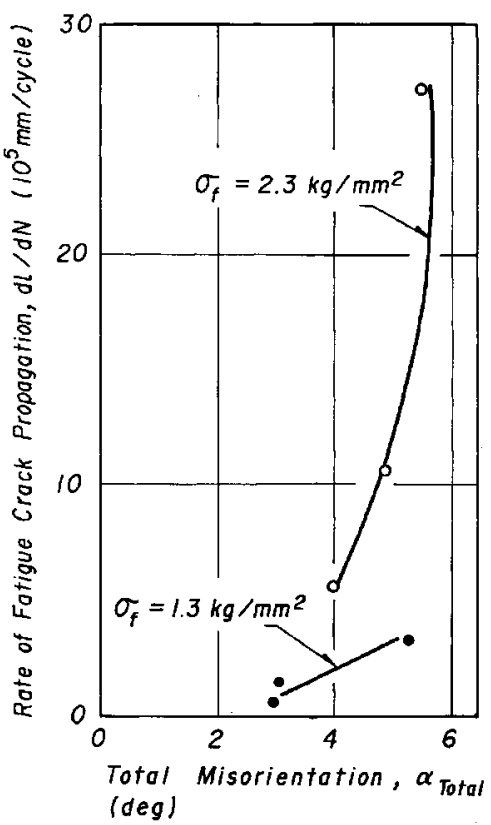

Fig. 19 Change in the propeagation rate of a fatigue crack, $d L / d N$, with the total misorientation, $\alpha_{\text {Total, }}$ at the tip of a fatigue crack.

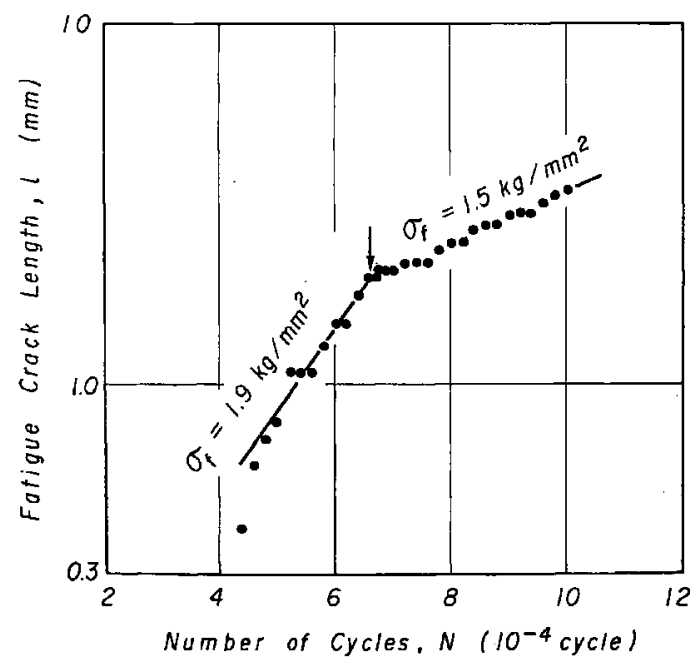

Fig. 20 :Change in the propagation rate of a fatigue crack, $d l / d N$, with decreasing fatigue stress, $\sigma_{f}$, from $1.9 \mathrm{~kg} / \mathrm{mm}^{2}$ to $1.5 \mathrm{~kg} / \mathrm{mm}^{2}$. An arrow shows the point where the fatigue stress was decreased.

crack and the structural parameters of the plastic zone at the crack tip.

As clearly seen in Figs. 16 19, the propagation rate of fatigue cracks is much larger in the ease of large stress fatigue even if the characteristics of the substructure are the same. The results suggest that contribution of the fatigue stress to the propagation rate of fatigue cracks should also be taken into consideration. A remarkable effect of the fatigue stress on the propagation rate of fatigue cracks is shown in Fig. 20. Although the characteristics of the substructure in the specimen immediately before and after the stress change are considered to be the same, the propagation rate of fatigue crack shows a sudden change due to the stress change. The results can also be explained by the proposed mechanism as follows. The multiplication rate 
of dislocations and the degree of stress concentration in the plastic zone may also vary due to the stress change. As a result, the changes in the generation rate of vacancies in the plastic zone and in the driving force for their diffusion will also occur. All the factors affect the propagation rate of fatigue crack.

From the results described above, it is concluded that the vacancy absorption mechanism for fatigue crack growth is consistent with the observed results.

\section{Summary and Conclusion}

Polycrystalline aluminium specimens were fatigued in alternate tension and compression with zero mean stress, in order to obtain a fundamental knowledge on the role of the substructure in fatigue crack propagation. During the course of fatigue crack propagation, some structural parameters and the size of the plastic zone formed around a fatigue crack were examined by the $\mathrm{X}$-ray microbeam technique. The propagation rate of fatigue cracks was also measured by a replicating method. The results obtained are summarized as follows :

(1) Regardless of the intensity of fatigue stress and the length of a fatigue crack, there exists a plastic zone around a fatigue crack in aluminium.
(2) A well-defined substructure is observed in the plastic zone. The substructure is characterized by the following three features : (i) heavily fragmented subgrains ( $1 \sim 2 \mu$ in diameter), (ii) very low excess dislocation density within a subgrain (about $10^{8} / \mathrm{cm}^{2}$ ), and (iii) sharply defined sub-boundaries.

(3) Two kinds of plastic zones, one observed by the $\mathrm{X}$-ray microbeam technique and the other found in the studies made from a macroscopic point of view, are concluded to be the same.

(4) Propagation rate of fatigue crack, $d l / d N$, can be related to the size of the plastic zone, $Z_{p}$, by the equation

$$
d l / d N=A Z_{p}^{m}
$$

where $A$ is a constant determined independently of fatigue stress, and $m$ the exponent of about 2 . It is shown that the same relation can be deduced from the studies from a macroscopic point of view. The fact also supports the preceding conclusion.

(5) Intimate relations exist between the propagation rate of a fatigue crack and some structural parameters of the plastic zone. The rate is also related to fatigue stress independently of the structural parameters. The results can be explained qualitatively by a vacancy absorption mechanism for the propagation of fatigue crack proposed by the present authors. 